\title{
Locus- and domain-dependent control of DNA methylation at mouse B1 retrotransposons during male germ cell development
}

\author{
Kenji Ichiyanagi, ${ }^{1,9}$ Yungfeng $\mathrm{Li}^{1}{ }^{1}$ Toshiaki Watanabe, ${ }^{2}$ Tomoko Ichiyanagi, $^{1}$ \\ Kei Fukuda, ${ }^{1,3}$ Junko Kitayama, ${ }^{4}$ Yasuhiro Yamamoto, ${ }^{1}$ Satomi Kuramochi-Miyagawa, ${ }^{5}$ \\ Toru Nakano, ${ }^{5}$ Yukihiro Yabuta, ${ }^{6,7}$ Yoshiyuki Seki, ${ }^{7,8}$ Mitinori Saitou, ${ }^{6,7}$ \\ and Hiroyuki Sasaki ${ }^{1}$ \\ ${ }^{1}$ Division of Epigenomics, Medical Institute of Bioregulation, Kyushu University, 3-1-1 Maidashi, Higashi-ku, Fukuoka 812-8582, \\ Japan; ${ }^{2}$ Department of Cell Biology, School of Medicine, Yale University, New Haven, Connecticut 06510, USA; ${ }^{3}$ Department \\ of Genetics, School of Life Science, The Graduate University for Advanced Studies (SOKENDAl), Mishima, Shizuoka 411-8540, \\ Japan; ${ }^{4}$ Division of Agricultural Genetics, National Institute of Genetics, Mishima, Sizuoka 411-8540, Japan; ${ }^{5}$ Department of \\ Pathology, Graduate School of Frontier Biosciences, Osaka University, Suita, Osaka 565-0871, Japan; ${ }^{6}$ Department of Anatomy \\ and Cell Biology, Graduate School of Medicine, Kyoto University, Kyoto 606-8501, Japan; ' Laboratory for Mammalian Germ Cell \\ Biology, Center for Developmental Biology, RIKEN Kobe Institute, Kobe, Hyogo 650-0047, Japan; ${ }^{8}$ Department of Bioscience, \\ School of Science and Technology, Kwansei Gakuin University, Sanda, Hyogo 669-1337, Japan
}

\begin{abstract}
In mammals, germ cells undergo striking dynamic changes in DNA methylation during their development. However, the dynamics and mode of methylation are poorly understood for short interspersed elements (SINEs) dispersed throughout the genome. We investigated the DNA methylation status of mouse BI SINEs in male germ cells at different developmental stages. Bl elements showed a large locus-to-locus variation in methylation; loci close to RNA polymerase II promoters were hypomethylated, while most others were hypermethylated. Interestingly, a mutation that eliminates Piwi-interacting RNAs (piRNAs), which are involved in methylation of long interspersed elements (LINEs), did not affect the level of BI methylation, implying a piRNA-independent mechanism. Methylation at Bl loci in SINE-poor genomic domains showed a higher dependency on the de novo DNA methyltransferase DNMT3A but not on DNMT3B, suggesting that DNMT3A plays a major role in methylation of these domains. We also found that many genes specifically expressed in the testis possess Bl elements in their promoters, suggesting the involvement of B1 methylation in transcriptional regulation. Taken altogether, our results not only reveal the dynamics and mode of SINE methylation but also suggest how the DNA methylation profile is created in the germline by a pair of DNA methyltransferases.
\end{abstract}

[Supplemental material is available for this article.]

In mammals, up to $\sim 50 \%$ of the genome comprises repetitive sequences (Lander et al. 2001; Waterston et al. 2002; Lindblad-Toh et al. 2005; Mikkelsen et al. 2005, 2007; Warren et al. 2008; Elsik et al. 2009), including long interspersed elements (LINEs), short interspersed elements (SINEs), and long terminal repeat (LTR) retrotransposons (Deininger and Batzer 2002; Deininger et al. 2003). The activities of LINEs and LTR retrotransposons, which can act as aggressive mutators, are suppressed by epigenetic mechanisms, such as DNA methylation, in both somatic and germ cells (Walsh et al. 1998; Bourc'his and Bestor 2004; Tsumura et al. 2006). DNA methylation at CG dinucleotides is a key epigenetic modification in mammals (Bird 2002) that is essentially heritable in somatic cells. In contrast, it is modifiable in early embryos and germ cells. Primordial germ cells (PGCs) undergo extensive erasure of methylation (Hajkova et al. 2002; Seki et al. 2005), and in males, prospermatogonia (gonocytes) acquire new methylation marks (Sasaki and Matsui 2008) (for stages during male germ cell development, see

\footnotetext{
${ }^{9}$ Corresponding author.

E-mail ichiyanagi@bioreg.kyushu-u.ac.jp.

Article published online before print. Article, supplemental material, and publication date are at http://www.genome.org/cgi/doi/10.1101/gr.123679.111.
}

Fig. 1A). The methylation levels of LINEs and LTR retrotransposons follow these global changes in male germ cell development (Lane et al. 2003; Lees-Murdock et al. 2003; Kato et al. 2007; Popp et al. 2010).

SINEs are 100-500 bp in length and have sequence features that are distinct from those of LINEs and LTR retrotransposons. For example, SINEs possess an RNA polymerase III (Pol III) promoter (Kramerov and Vassetzky 2005), whereas LINEs and LTR retrotransposons possess a RNA polymerase II (Pol II) promoter. The mouse genome contains about 1.5 million SINE copies, which together constitute $8 \%$ of this genome (Waterston et al. 2002). Mouse SINEs are classified into families of B1, B2, B3, B4, MIR, and ID. B1 and B2, the largest families with 570,000 and 380,000 copies, respectively, contain currently active copies.

B1 is an $~ 150$-bp element in rodents that shares a common origin with Alu SINEs in primates. Interestingly, although B1 and Alu SINEs have independent evolutionary histories, they are both accumulated in similar gene-rich regions of the respective genomes, suggesting their roles in gene regulation (Lander et al. 2001; Waterston et al. 2002; Polak and Domany 2006; Tsirigos and Rigoutsos 2009). Analyses of methylation of the bulk of B1 copies have revealed that B1 elements are hypomethylated in PGCs and 


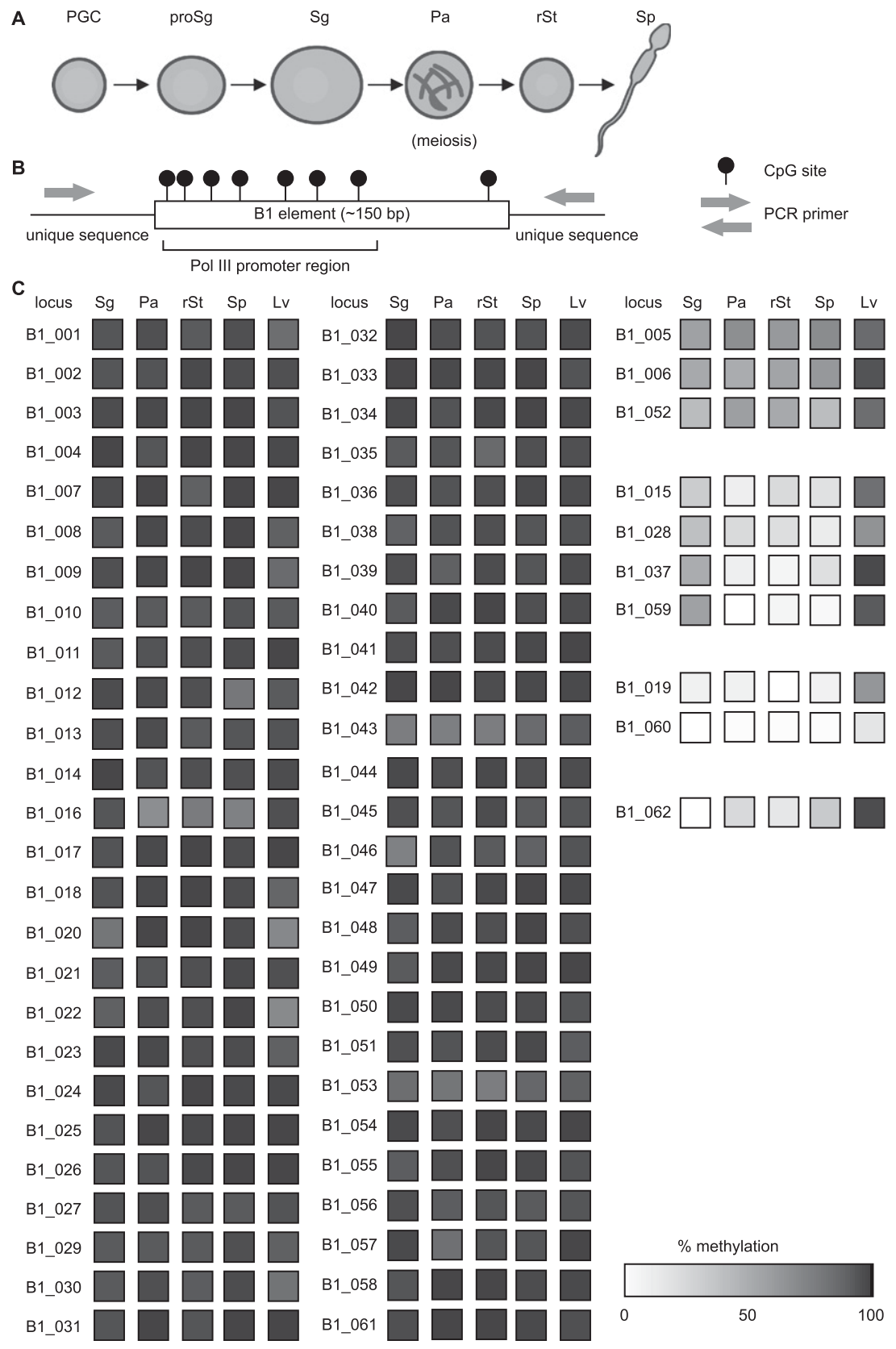

Figure 1. DNA methylation at $B 1$ loci in germ and somatic cells. $(A)$ Schematic representation of male germ cell development. (PGC) Primordial germ cell; (proSg) prospermatogonium; (Sg) spermatogonium; (Pa) pachytene spermatocyte; ( $\mathrm{rSt}$ ) round spermatid; (Sp) spermatozoon. (B) Experimental protocol for analysis of individual B1 elements. B1 elements (open rectangle) containing six to eight $\mathrm{CpG}$ sites (shown as lollipops) were selected, and primers (gray arrows) were designed for unique flanking regions. (C) Methylation of individual B1 elements. Methylation levels in the indicated cells (noted above the columns) at B1 loci designated on the left (B1_00-B1_062) are represented as filled squares on the basis of a grayscale: from white $=0 \%$ methylation to black $=100 \%$ methylation. (Lv) Liver cells.

hypermethylated in late prospermatogonia, spermatozoa, and somatic cells (Kato et al. 2007; Meissner et al. 2008; Popp et al. 2010).

Mammals have two DNA methyltransferases, DNMT3A and DNMT3B, that are responsible for de novo methylation and a regulatory protein with no catalytic activity, DNMT3L (Bestor 2000). Mutational studies in mice revealed that the two enzymes have redundant activities for de novo methylation of L1 (a member of LINEs) and intracisternal A particle (IAP, a member of LTR retro- transposons), whereas DNMT3L is critical for de novo methylation of both elements (Walsh et al. 1998; Bourc'his and Bestor 2004; Kato et al. 2007). Methylation of L1 and IAP has been recently proposed to be guided by a class of germ cell-specific small RNAs, designated Piwi-interacting RNAs (piRNAs), which are produced by the actions of PIWIL2 (also called MILI), PIWIL4 (MIWI2), DDX4 (MVH), MOV10L1, and PLD6 (MITOPLD) (Aravin et al. 2007, 2008; Carmell et al. 2007; Kuramochi-Miyagawa et al. 2008, 2010; Zheng et al. 2010; Watanabe et al. 2011a). Tudor-domain proteins, TDRD1 and TDRD9, and GASZ are also involved in piRNA biogenesis and de novo L1 methylation in male germ cells (Ma et al. 2009; Reuter et al. 2009; Shoji et al. 2009). The piRNA-dependent DNA methylation in mammals is analogous to the RNA-directed sequence-specific DNA methylation in higher plants (Matzke et al. 2007). However, the generality of the piRNAdependent DNA methylation remains unclear because only a few retrotransposons have been analyzed until this date.

Here we analyzed the DNA methylation status of a number of individual B1 loci in male germ cells at various developmental stages as well as in spermatogonia deficient in de novo methylation or piRNA biogenesis. Our results revealed that most loci were stably hypermethylated during spermatogenesis, with some variations dependent on the locus and domain. By incorporating these results from both wild-type and mutant germ cells, we propose a model that explains how de novo methylation of $\mathrm{B} 1$ is regulated in the germ cell genome. Moreover, we provide data that suggest a role for B1 in transcriptional regulation of testis-specific genes and discuss the impact of B1 retrotransposition in rodent evolution.

\section{Results}

Locus-dependent variations in B1 methylation in male germ cells

The consensus sequence of young B1 subfamilies has eight CG sites, most of which reside in the Pol III promoter region (Fig. 1B). Previous studies (Kato et al. 2007; Popp et al. 2010) have reported on the DNA methylation status of the bulk of genomic B1 copies in some somatic tissues and germ cells, providing rough estimates of their overall methylation levels. However, the methylation levels were most likely underestimated because of the occurrence of frequent CG-to-TG mutations (unmethylated CG and TG are indistinguishable in bisulfite sequencing readouts) (see legend for Supplemental Fig. S1). More importantly, the extent of var- 
iation in methylation levels among individual loci remains unknown.

We analyzed 62 individual B1 elements that were selected on the basis of their size, sequence conservation, and number of CG sites (Fig. 1C; see Methods for the detailed selection method). In liver cells (a somatic control), almost all loci examined were uniformly hypermethylated with an average methylation level of 92\%, with only one exception (B1_060; 16\% methylation). In spermatogenic cells, most loci $(n=52)$ showed stable hypermethylation ( $>80 \%$ methylation), while 10 loci showed lower levels of methylation. Of these 10 loci, three (B1_005, B1_006, and B1_052) showed intermediate levels of methylation (40\%-70\%), and three others (B1_019, B1_060, and B1_062) showed very low or virtually no methylation throughout spermatogenesis. The remaining four (B1_015, B1_028, B1_037, and B1_059) showed intermediate levels of methylation in spermatogonia and became hypomethylated during (spermatocytes) and after meiosis (spermatids and spermatozoa). Taken together, male germ cells, particularly those at later spermatogenic stages, exhibited significantly less methylation at some B1 loci compared with liver cells.

The existence of some hypomethylated loci in male germ cells prompted us to examine the expression levels of $\mathrm{B} 1$ RNA in the testis. Consistent with a previous study (Li et al. 1999), B1 RNA of $\sim 150$ nt was accumulated in the testis but not in other tissues such as the brain, heart, lung, intestine, liver, or kidney (Supplemental Fig. S2A). We infer that hypomethylated loci may be the source of testis-accumulated B1 RNA, but a high similarity in nucleotide sequence among young B1 elements precluded detailed analysis of locus-specific B1 transcription. We also found that B1 elements were poorly methylated in oocytes (Supplemental Fig. S1), wherein B1 expression is very high (Kaplan et al. 1985; Peaston et al. 2004). These results are consistent with the inverse correlation between DNA methylation and Pol III promoter activity.

\section{B1 methylation is independent of piRNA production}

We next analyzed the methylation status of the bulk of B1 elements in male germ cells (Supplemental Fig. S1). These results suggest that de novo methylation of B1 occurred between embryonic day 12.5 (E12.5; PGCs) and postnatal day 0 (P0; prospermatogonia). Because de novo methylation at L1 and IAP involves prospermatogonial piRNAs (Aravin et al. 2007, 2008; Kuramochi-Miyagawa et al.

$\mathbf{E}$
2008, 2010; Watanabe et al. 2011a), we analyzed the previously published expression profiles of small RNAs (24-31 nt in length) in E16.5 (fetal) and P10 (prepuberal) testes (Aravin et al. 2007, 2008).

B1-derived piRNAs corresponding to various portions of the B1 sequence were found in the E16.5 library (Fig. 2A). Importantly, a substantial fraction of the sense and antisense pairs had a 10-bp complementary sequence (Fig. 2C), and the majority had uracil at the first position and adenine at the tenth position (Fig. 2E); these are the signatures of piRNAs produced via the ping-pong cycle (Brennecke et al. 2007; Gunawardane et al. 2007). In P10 testes, where spermatogonia are the predominant cell type, the number
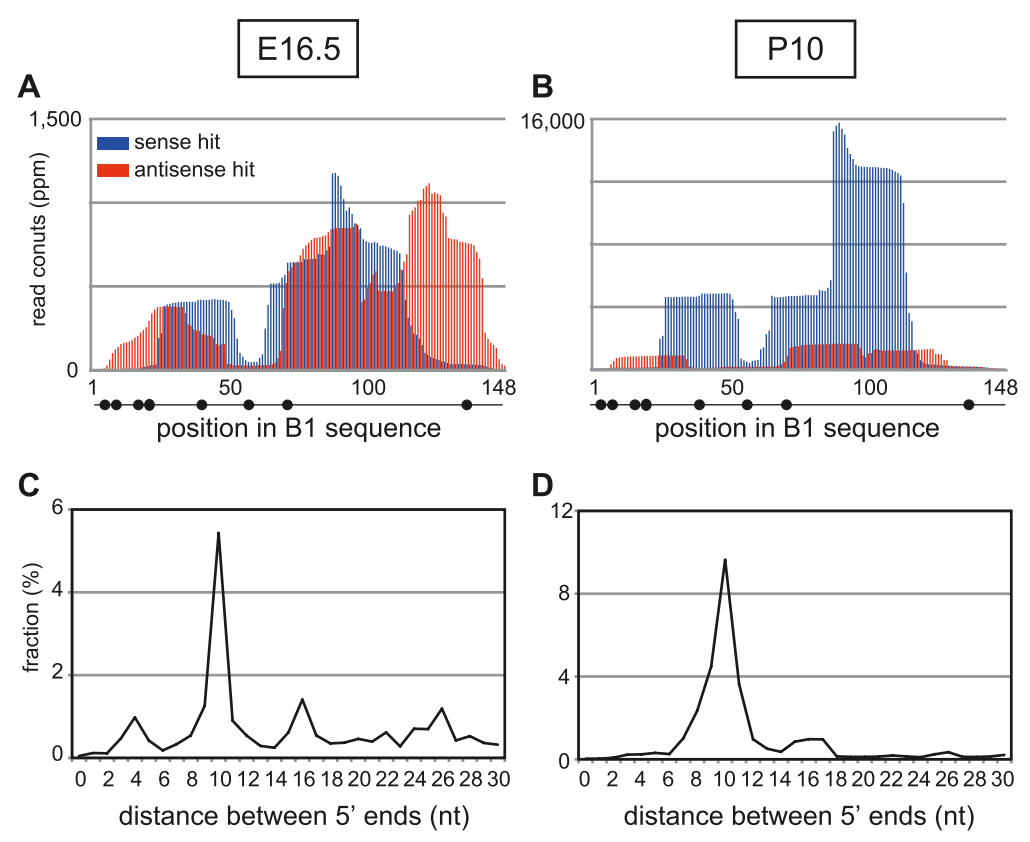

D
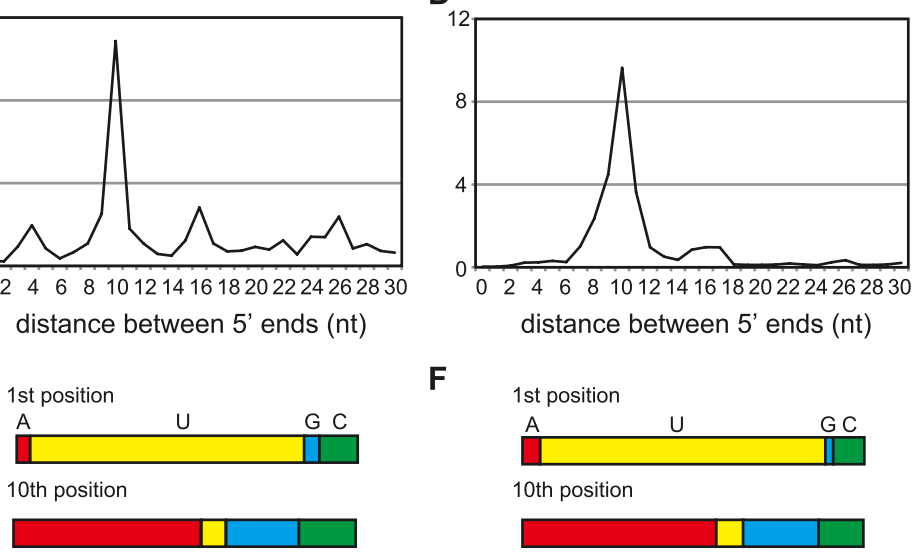

$\mathbf{F}$
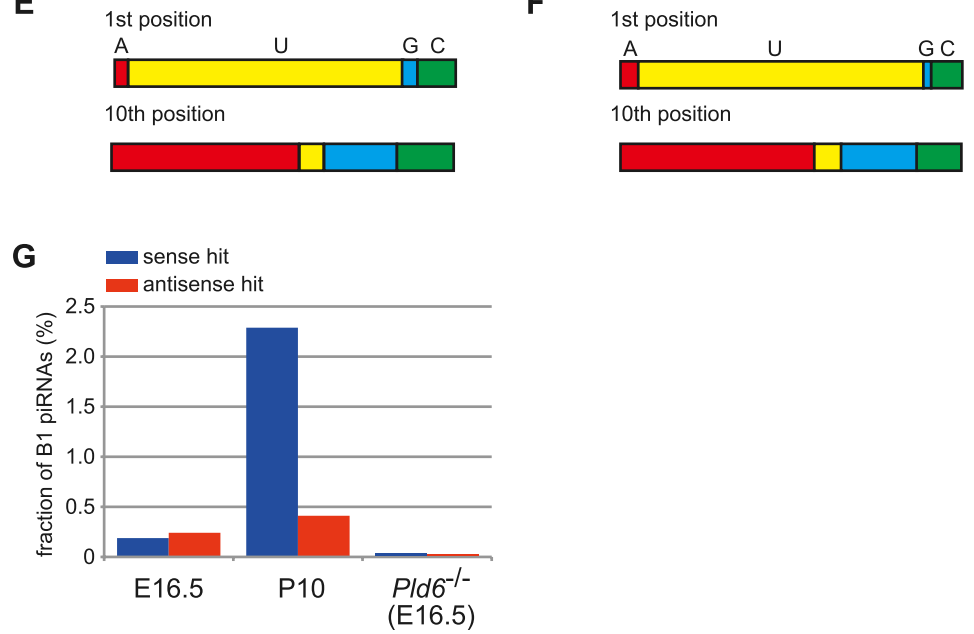

Figure 2. Analysis of B1-derived small RNAs in fetal and prepuberal testes. Small RNAs in E16.5 (Aravin et al. 2008) (ACE) and P10 (Aravin et al. 2007) (BDF) testes were mapped to B1_Mus1 consensus sequences. $(A, B)$ Relative read counts (number of hits divided by the total sequence reads, expressed as parts per million, ppm) of sense (blue) and antisense (red) small RNAs. (C,D) The start position of each sense-strand read was compared with that of all antisense-strand reads. The percentages of relative length overlap between sense and antisense reads are shown. $(E, F)$ Nucleotide compositions at the first and tenth positions of B1-derived small RNAs. (G) The relative abundance of B1-derived small RNAs. The fractions of sense (blue) and antisense (red) reads in the total sequence reads are shown. The data for piRNAs in PId6/MitoPLD KO prospermatogonia were obtained from Watanabe et al. (2011a).

\section{Genome Research www.genome.org}


of B1-derived sense piRNAs increased by $>10$-fold, whereas the number of antisense piRNAs increased only slightly (Fig. 2B,G). These piRNAs also showed the signatures of ping-pong cycle products (Fig. 2D,F).

The presence of B1-derived piRNAs in prospermatogonia raised the possibility of these piRNAs playing a role in de novo methylation of B1 elements, similar to that in de novo methylation of L1 and IAP elements. Therefore, we analyzed the B1 methylation status in spermatogonia from Pld6 knockout (KO) mice in whom prospermatogonial piRNAs were markedly decreased (Fig. 2G; Watanabe et al. 2011a). The methylation levels at the B1 loci analyzed were largely unaffected (Fig. 3A,E). Further analysis on selected B1 loci in wild-type, Pld6 KO, and Piwil2 KO prospermatogonia revealed no significant decrease in $\mathrm{B} 1$ methylation in these mutant
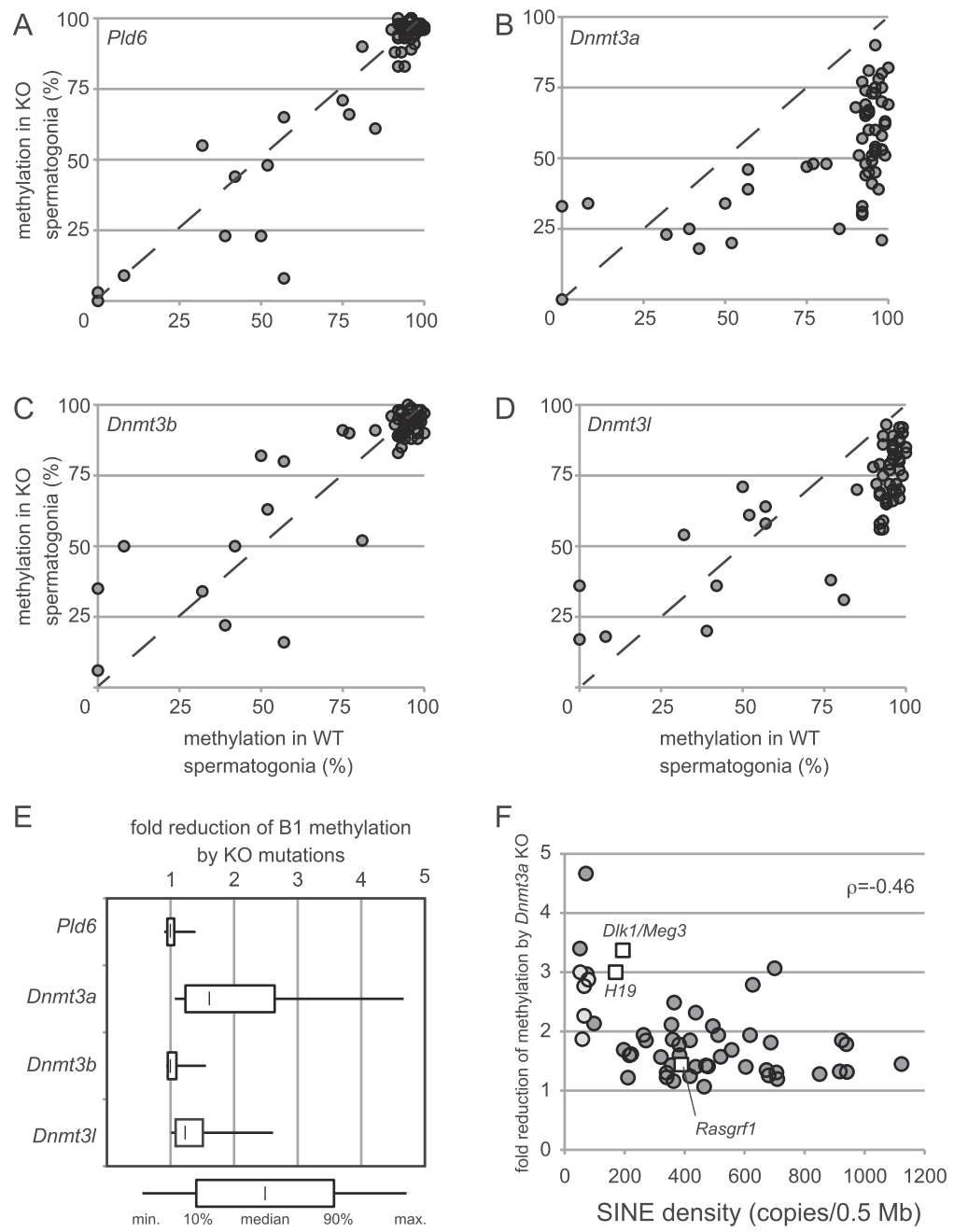

Figure 3. Effects of mutations in piRNA biogenesis and DNA methyltransferases on de novo B1 methylation. Methylation levels ( $y$-axis) at individual B1 loci in spermatogonia of Pld6 $6^{-1-}(A)$, Dnmt3 $a^{\text {2lox/1lox }}$ TNAP-Cre $(B)$, Dnmt3 $b^{2 \text { lox/1lox } T N A P-C r e ~}(C)$, and Dnmt $3 I^{-1-}(D)$ are plotted against their methylation levels in wild-type spermatogonia ( $x$-axis). Dashed lines show the $y=x$ slope. $(E)$ Fold reductions in individual B1 methylation due to mutations. Only the data for B1 loci with $\geq 80 \%$ methylation in wild-type spermatogonia are included. $(F)$ Fold reductions in methylation due to Dnmt3a $\mathrm{KO}$ mutation at individual B1 loci (dark gray circles) are plotted against the SINE density in the flanking genomic regions ( $0.25 \mathrm{Mb}$ of each side). B1 loci selected from SINE-poor domains (B1_101-B1_105) are shown as light gray circles. The methylation levels at three paternally methylated regions for genomic imprinting (H19, Dlk1/Meg3, and Rasgrf1) in wild-type and Dnmt3a KO spermatogonia were examined as described previously (Kato et al. 2007), and the results are shown as open squares. Spearman's correlation coefficient $(\rho)$ is shown on the right. cells (Supplemental Fig. S3). These results indicate that B1 elements could be de novo methylated in prospermatogonia independent of the piRNA pathway. Moreover, the accumulation of full-length B1 RNA in spermatogonia was not increased by the Pld6 piRNAs have little effect on DNA methylation and RNA stability of because DICER-dependent endogenous siRNAs of B1 origin regulate We monomers of L1 was markedly affected in the Pld6 KO spermatogonia (Supplemental Fig. S4). In stark contrast, methylation of the bulk of IAP and MMERVK10C elements, whose piRNAs are abundant in prospermatogonia (KuramochiMiyagawa et al. 2008), was largely unaffected (Supplemental Fig. S4), although a previous report showed that specific copies of an IAP subtype were affected in piRNA pathway mutants (KuramochiMiyagawa et al. 2008, 2010; Watanabe et al. 2011a). Thus, even for autonomous retrotransposons, different elements show different levels of piRNA dependency for targeted DNA methylation.

\section{Dependence of $\mathrm{B} 1$ methylation on Dnmt3a, Dnmt3b, and Dnmt3I}

To understand which DNMT3 family proteins were involved in de novo methylation of B1, we examined methylation levels at B1 loci in spermatogonia from conditional KO mutants for Dnmt3a (Dnmt3a $a^{2 \text { lox/1lox }}$, TNAP-Cre) and Dnmt3b (Dnmt3b $b^{2 \text { lox/1lox }}$ TNAP-Cre), as well as those from conventional Dnmt $3 \mathrm{l}$ KO mice. In conditional KO mice, Dnmt $3 a$ and Dnmt $3 b$ are inactivated in germ cells before the prospermatogonial stage (Kaneda et al. 2004; Kato et al. 2007).

At most B1 loci examined, the methylation level was substantially reduced in Dnmt3a KO spermatogonia (Fig. 3B,E) but not in Dnmt3b KO spermatogonia (Fig. 3C,E). Dnmt3l KO spermatogonia showed methylation that was reduced to an intermediate level (Fig. 3D,E). These results are consistent with the fact that DNMT3A cooperates with DNMT3L for de novo methylation in germ cells, but the milder defect in Dnmt3l KO spermatogonia suggests that DNMT3A also acts independent of DNMT3L. This is in contrast with previous findings on IAP and L1 elements, where Dnmt3l KO severely affected methylation but single Dnmt3a or Dnmt3b KO did not (Bourc'his and Bestor 2004; Kato et al. 2007).

Of note, the effect of Dnmt3a KO on methylation varied among the B1 loci, ranging from almost no change to about 
a fivefold reduction (Fig. 3E). This suggests that although Dnmt3b KO did not affect B1 methylation, Dnmt $3 b$ may have a back-up role. We explored the genomic features that affected the dependence on Dnmt3a and found that methylation tended to be more Dnmt3a-dependent if the B1 locus was in a SINE-poor, LINErich, gene-poor, or low $\mathrm{G}+\mathrm{C}$ genomic region (Fig. 3F; Supplemental Fig. S5). Among these features, SINE density showed the highest correlation to Dnmt3a dependency (Spearman's $\rho=-0.46,+0.39$, -0.27 , and -0.17 for Dnmt3a dependency vs. SINE density, LINE density, gene density, and $\mathrm{G}+\mathrm{C}$ content, respectively). To confirm this correlation, we selected an additional five B1 loci (B1_101B1_105) that were present in SINE-poor regions $(<100$ SINEs/0.5 $\mathrm{Mb})$ and examined their methylation levels in wild-type and Dnmt3a KO spermatogonia. These loci indeed showed high levels of Dnmt3a dependency (Fig. 3F). Interestingly, three paternally methylated regions from the imprinted loci also followed the same rules (Fig. 3F). De novo methylation of the $H 19$ and Dlk1/Meg3 differentially methylated regions (DMRs) were highly dependent on Dnmt3a; these DMRs were located in SINE-poor regions. De novo methylation of the Rasgrf1 DMR did not depend on Dnmt3a; this DMR was located outside the SINE-poor regions. Interestingly, SINE-poor domains were not distributed evenly within the nucleus; fluorescence in situ hybridization (FISH) using SINE (B1 plus B2) and LINE (L1) probes showed that SINE-poor domains tended to be located at the periphery of prospermatogonial nuclei (Fig. 4; Supplemental Fig. S6). These domain-dependent distribution patterns might be involved in the observed variations in Dnmt3a dependency (see Discussion).

\section{B1 loci in Pol II promoter regions are protected from methylation}

As described above, B1 loci showed large differences in methylation in wild-type germ cells despite the fact that the internal nucleotide sequences did not significantly differ between the hyperand hypomethylated loci (Supplemental Fig. S7). We explored what genomic features might be associated with the hypomethylated loci. The local LINE, SINE, and gene densities, G+C content, and the location in terms of the Pol II transcription unit (i.e., genic
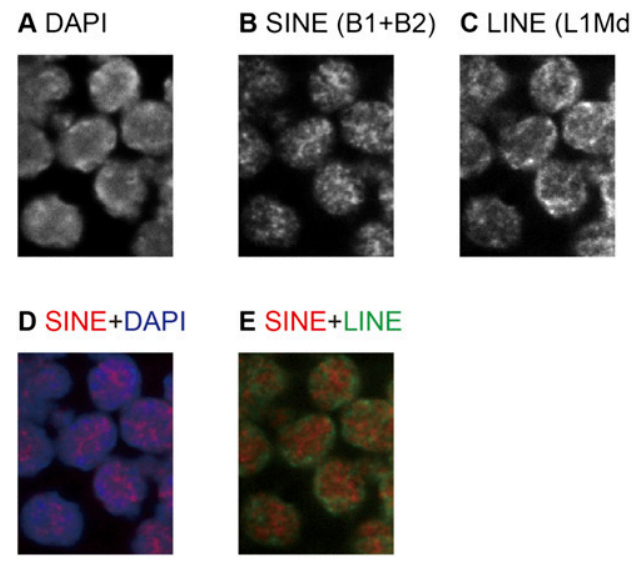

Figure 4. Spatial distributions of SINE and LINE elements in prospermatogonial nuclei. Frozen sections of E15.5 testis were stained with 4',6-diamidino-2-phenylindole (DAPI) $(A)$ and hybridized against Cy3dUTP-labeled B1/B2 probes $(B)$ and green dUTP-labeled L1 probes $(C) .(D)$ Merged image of panels $A$ and $B$. (Red) SINE; (blue) DAPI. (E) Merged image of panels $B$ and $C$. (Red) SINE; (green) LINE. Images of non-adhering germ cells in a seminiferous tubule are shown (600× magnification). vs. intergenic) were not major factors contributing to protection from methylation (Supplemental Fig. S8A-E). However, we found that B1 loci located at $<1 \mathrm{~kb}$ from a transcription start site (TSS) were generally hypomethylated, regardless of their orientation relative to the transcript (Fig. 5A). The CpG density of the neighboring regions was also correlated (Supplemental Fig. S8F), which may have reflected the CpG-rich nature of the regions around TSSs.

To confirm this correlation, we randomly selected an additional 16 B1 loci (B1_201-B1_216) located within $1 \mathrm{~kb}$ from TSS and analyzed their methylation levels in spermatogenic cells. Most of these loci were hypomethylated in spermatogenic cells (Fig. 5B). It is noteworthy that some loci (B1_203, B1_206, B1_208, B1_209, B1_211, B1_028, B1_215, B1_216, and B1_059) were moderately (17\%-58\%) methylated in spermatogonia and then lost methylation in pachytene spermatocytes. Overall, promoter-proximal B1 loci were significantly less methylated than other loci $\left(p<10^{-8}\right)$ (Fig. $5 \mathrm{C})$. This trend was more evident in germ cells because B1s located at $>0.3 \mathrm{~kb}$ away from TSSs showed denser methylation in liver cells than in germ cells (Fig. 5B). The higher methylation levels at these B1 loci in liver cells were not correlated with the methylation levels at the TSS regions themselves because these regions were consistently unmethylated in all cells examined (Supplemental Fig. S9).

In general, the density of retrotransposons is extremely low in Pol II promoter regions (Fig. 6A). Although this is also true for B1 elements, we found that they were at a higher density in testisspecific promoters compared with all promoters (Fig. 6A,B; $p<10^{-16}$ by $\chi^{2}$ test). These results suggest the possibility that the presence of a B1 element in the proximity of TSS is associated with suppression of genes in somatic cells. Furthermore, differential methylation of TSS-proximal B1 elements between germ and somatic cells, as shown above, may be involved in regulation of these genes.

To examine these possibilities, we compared the expression levels of genes possessing B1 elements in their TSS-proximal regions in spermatogonia and liver cells (Fig. 6C). Almost all genes examined showed elevated expression in spermatogonia over that in liver cells. In addition, the difference in expression was larger if the neighboring B1 was hypomethylated in spermatogonia but hypermethylated in liver cells (Pearson's $r=0.49$ for differences in expression and neighboring B1 methylation). These results are consistent with a role for B1 elements in testis-specific gene expression.

\section{Discussion}

We examined the methylation levels at a panel of B1 loci in male germ cells at various developmental stages and in somatic tissue. In adult liver cells, most B1 loci were hypermethylated, consistent with the lack of B1 expression, although a small fraction located close to a Pol II promoter showed relatively lower methylation. Because human Alu elements show similar hypomethylation status around Pol II TSSs and in CpG-rich regions in somatic cells (Rodriguez et al. 2008; Xie et al. 2009; Edwards et al. 2010), this may be a common feature of mammalian SINEs. We found that this hypomethylation tendency of TSS-proximal B1 elements was much more evident in germ cells, especially during and after meiosis. Moreover, we showed that these B1-associated genes were highly expressed in germ cells, and, conversely, testis-specific TSS regions possess significantly more B1 elements than other TSS regions. These results suggest the possible involvement of B1 elements in regulation of testis-specific genes. B1 elements may play a role in stably silencing Pol II promoters in somatic cells, perhaps partly through methylation. Thus, a retrotranspositional insertion of a B1 element into a promoter region could modulate tissue-specific gene expression. 
SINE methylation during male germ cell development

A
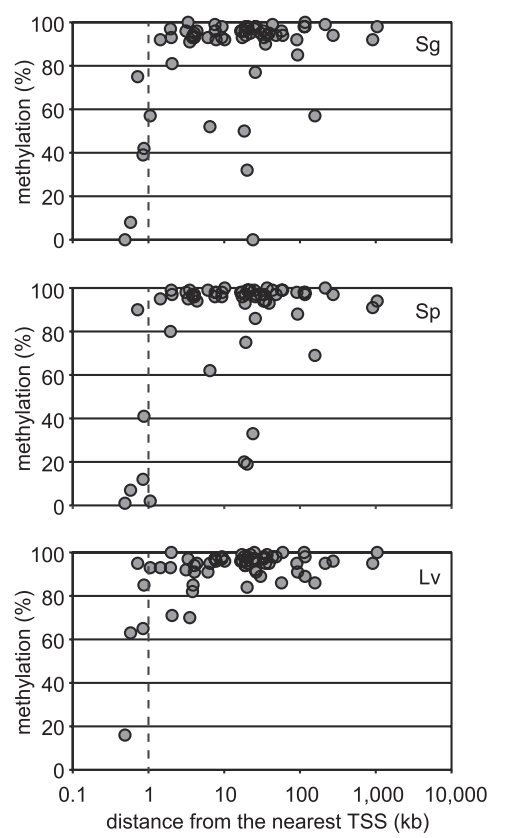

C
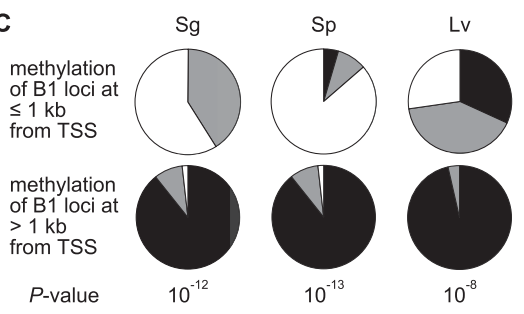

Figure 5. DNA methylation levels and proximity to TSSs. (A) Methylation levels at individual B1 loci $(y$-axis) are plotted against their distances to the nearest TSS ( $x$-axis). Results for spermatogonia, spermatozoa, and liver are shown. Results for pachytene spermatocytes and round spermatids were nearly the same as those for spermatozoa (see Fig. 1C). (B) Methylation levels at B1 loci (B1_201-B1_216) that are present at $<1 \mathrm{~kb}$ from TSS are shown on a grayscale as in Figure 1C. TSS-proximal B1 loci in the initial group (B1_060, B1_019, B1_046, B1_028, B1_052, and B1_059) are also included. (C) Statistics for B1 methylation and their distances from TSS. The $78 \mathrm{~B} 1$ loci $(62+16)$ are categorized into those at $\leq 1 \mathrm{~kb}$ from TSS (upper pie charts) and those at $>1 \mathrm{~kb}$ (lower pie charts). The loci are further categorized into 0\%-19\% methylation (white), 20\%-79\% methylation (gray), and 80\%-100\% methylation (black). The $P$-values from $\chi^{2}$ tests are shown at the bottom.

Because B1 is retrotranspositionally active, this process may be ongoing in rodent evolution. In addition, the retrotransposed copies could then be another source of retrotransposition because of their tendency to be hypomethylated in germ cells.

We also showed that B1 loci in non-promoter regions were generally hypermethylated in spermatogenic cells. Our study with Pld6 and Piwil2 KO mice revealed that de novo methylation of B1 elements in prospermatogonia was largely independent of the piRNA pathway. This is in contrast to the piRNA-dependent de novo methylation of L1 elements (Aravin et al. 2007; Carmell et al. 2007; Kuramochi-Miyagawa et al. 2008, 2010; Watanabe et al. 2011a). Interestingly, methylation of the bulk of IAP elements was largely unaffected in Pld6 KO spermatogonia, although previous reports showed that two specific IAP loci were significantly affected in Pld6, Piwil2, and Piwil4 KO germ cells (Kuramochi-Miyagawa et al. 2008; Watanabe et al. 2011a). We also found that methylation of the bulk of MMERVK10C elements was unaffected, indicating that the involvement of the piRNA pathway in methylation was dependent on the retrotransposon class. Of note, the promoter of mouse L1 elements contains tandem repeats, whereas the promoters of IAP, MMERVK10C, and B1 elements do not. Therefore, it is conceivable that piRNAs (or processes generating piRNAs) play a more important role in targeted methylation of retrotransposon promoters consisting of tandem repeats of CpG-rich sequences. Consistent with this idea is the fact that the two IAP loci at which methylation depends highly on piRNAs have a specific segmental duplication of 80 bp (i.e., tandem repeat), which is absent from most IAP copies (data not shown). Moreover, the promoter of a non-coding RNA in the Rasgrf1 locus consists of CpG-rich tandem repeats, and its de novo methylation is highly dependent on piRNAs (Watanabe et al. 2011b), which also supports this idea.

In the mouse, de novo DNA methylation is catalyzed by DNMT3A and DNMT3B, and DNMT3L plays a role in assisting these two enzymes specifically in germ cells. Dnmt $3 l$ is indispensable for de novo methylation of the paternally methylated DMRs as well as the promoters of L1 and IAP retrotransposons (Bourc'his and Bestor 2004; Kaneda et al. 2004; Kato et al. 2007). Methylation of the $H 19$ and Dlk1/Meg3 DMRs depends on the DNMT3A enzyme, whereas DNMT3A and DNMT3B have redundant functions in methylation of the Rasgrf1 DMR (Kaneda et al. 2004; Kato et al. 2007). To date, the mechanism underlying this discrepancy remains unknown.

Our analysis of B1 methylation revealed that methylation of the loci located in SINE-poor domains was more dependent on Dnmt3a, suggesting that its principal role is in methylation of SINE-poor genomic domains, which include $H 19$ and Dlk1/Meg3, but not Rasgrf1. However, there remains a caveat. Methylation of the bulk of L1 elements enriched in SINE-poor regions did not show a significant Dnmt3a dependency. We speculate that the piRNAs assist DNMT3A and DNMT3B enzymes in methylating L1 elements regardless of their genomic locations.

SINEs are not distributed evenly along the chromosome arms. Rather, mammalian chromosomes consist of alternating SINErich/LINE-poor and LINE-rich/SINE-poor domains (Supplemental Fig. S6A), and SINE-poor genomic regions often coincide with chromosomal $\mathrm{G}$ bands, low $\mathrm{G}+\mathrm{C}$ isochors, and gene-poor regions (Lander et al. 2001; Waterston et al. 2002). Among these genomic variables, we showed that the SINE density was most relevant to the observed variations in Dnmt3a dependency. We showed that SINE-poor/LINE-rich genomic regions were not spatially dispersed. Rather, they were localized at the periphery of prospermatogonial nuclei, as reported for somatic nuclei of humans and mice (Bolzer et al. 2005; Guelen et al. 2008; Solovei et al. 2009). Thus, an intriguing speculation is that DNMT3A can efficiently methylate 
A

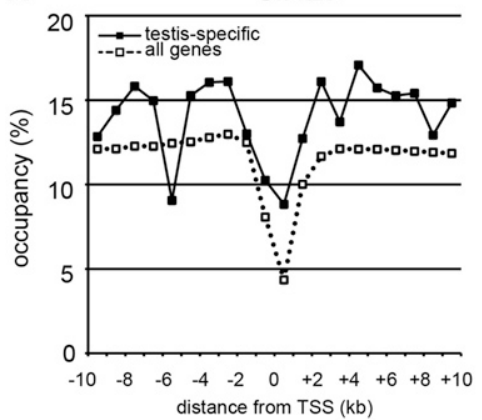

B

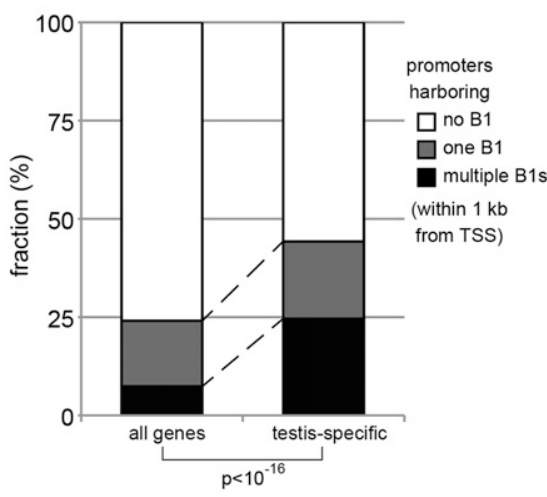

LINEs

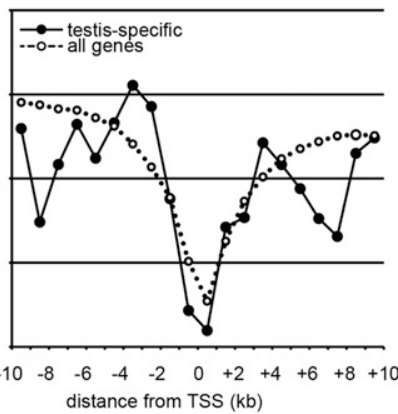

C

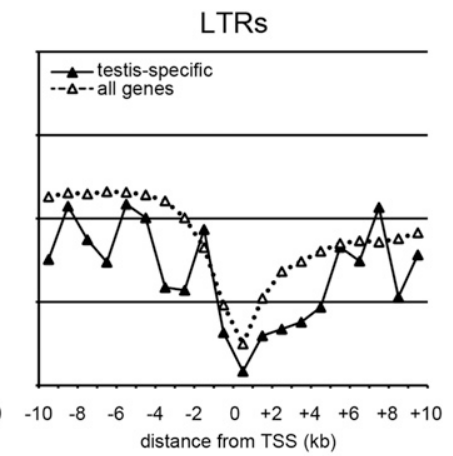

\begin{tabular}{|c|c|c|c|c|c|}
\hline \multirow[b]{2}{*}{ B1_locus } & \multicolumn{3}{|c|}{ B1 methylation (\%) } & \multirow[b]{2}{*}{ gene } & \multirow{2}{*}{$\begin{array}{l}\text { mRNA diff. } \\
\text { (ratio of } S g \text { to } L v \text { ) }\end{array}$} \\
\hline & $\mathrm{Sg}$ & Lv & diff. & & \\
\hline B1_216 & 22 & 96 & 74 & AK054519 & 109 \\
\hline B1_214 & 12 & 78 & 66 & Nid1 & 22 \\
\hline B1_209 & 18 & 81 & 63 & Polr3h & 21 \\
\hline B1_211 & 21 & 81 & 60 & Zfp759 & 3.2 \\
\hline B1_203 & 17 & 67 & 50 & Arpc3 & 4.1 \\
\hline B1_052 & 42 & 85 & 43 & BC023829 & 8.5 \\
\hline B1_207 & 11 & 52 & 41 & Dhdh & 0.4 \\
\hline B1_059 & 57 & 93 & 36 & Mbtps2 & 5.2 \\
\hline B1_206 & 0 & 34 & 34 & Ccar1 & 22 \\
\hline B1_028 & 39 & 65 & 26 & Eif3c & 16 \\
\hline B1_210 & 14 & 33 & 19 & Polr2k & 6.1 \\
\hline B1_212 & 2 & 17 & 15 & $A t / 3$ & 11 \\
\hline B1_215 & 58 & 63 & 5 & Faf2 & 9.1 \\
\hline B1_205 & 0 & 3 & 3 & 1600002K03Rik & 8.3 \\
\hline B1_201 & 1 & 0 & -1 & Rpf1 & 6.1 \\
\hline
\end{tabular}

Figure 6. B1 methylation levels and gene expression. (A) Statistics for genomic occupancy of SINEs (squre), LINEs (circle), and LTR retrotransposons (triangle) in 1-kb bins are shown in relation to their distance from TSS for all (dashed lines) and testis-specific genes (solid lines). Testis-specific genes were listed previously (Weber et al. 2007). (B) Statistics for all and testis-specific genes that harbor 0 (white), 1 (gray), and >1 (black) B1 elements within 1 kb from TSS. (C) DNA methylation levels at B1 loci and expression levels of their neighboring genes. For each gene adjacent to a B1 locus, the relative proportion of mRNA levels in spermatogonia and liver (three biological replicates) was determined by quantitative RT-PCR.

the genomic DNA localized at the nuclear periphery, whereas DNMT3B cannot, although this is not the only possibility. In any event, our results shed light on the importance of discrete chromosomal domains for regulation of de novo methylation activities during male germ cell development.

\section{Methods}

\section{Selection of B1 loci for methylation analysis}

The RepeatMasker output and genome sequence were downloaded from the UCSC Genome Browser (http://www.genome.ucsc.edu/). We first selected B1 copies annotated as B1_Mm, B1_Mus1, or B1_Mus2, which were $\geq 135 \mathrm{bp}$, showed $<9 \%$ divergence from the respective consensus sequences, and possessed $\geq 6 \mathrm{CpG}$ sites. From this pool (consisting of $\sim 15,000$ copies), we randomly selected loci (B1_001-B1_062). B1 loci in SINE-poor domains (0.5-Mb regions harboring <100 SINE elements; B1_101-B1_105) and promoterproximal loci (at <1 kb from TSS; B1_201-B1_216) were randomly selected from the same pool. Their genomic locations are listed in Supplemental Table S1.

\section{Cell preparation}

Liver cells and spermatozoa were prepared separately from adult male C57BL/6J mice. Spermatogonia were isolated from P7 testes as follows: Seminiferous tubules were dissociated from testes by incubating in PBS containing $0.25 \%$ trypsin and $10 \mathrm{mM}$ EDTA for
$10 \mathrm{~min}$ at $37^{\circ} \mathrm{C}$. The cell suspension was passed through a $40-\mu \mathrm{m}$ nylon mesh and washed with PBS containing 10\% fetal bovine serum (FBS). The cells were incubated with anti-EpCAM antibody (Santa Cruz Biotechnology) and Alexa Fluor 488 goat anti-rat IgG (Invitrogen), and the labeled cells were collected using FACS JSAN (Bay Bioscience). We confirmed that $>95 \%$ of the sorted cells were positive for CD9 (BD Biosciences), another cell surface marker for spermatogonia. Pachytene spermatocytes and round spermatids were harvested from 5-wk-old testes. Seminiferous tubules were dissociated by incubating for $15 \mathrm{~min}$ at room temperature in PBS containing $50 \mathrm{U} / \mathrm{mL}$ of collagenase (Sigma), followed by incubation in PBS containing $0.05 \%$ trypsin for $20 \mathrm{~min}$ at $37^{\circ} \mathrm{C}$. The cell suspension was then passed through a $40-\mu$ m nylon mesh and washed with PBS containing 10\% FBS. After incubation in PBS containing $5 \mathrm{mM}$ Vybrant DyeCycle Ruby stain (Invitrogen) and $10 \%$ FBS, the cells were sorted on the basis of their fluorescence emission intensity, which was correlated with the DNA content, to collect pachytene spermatocytes $(4 n)$ and round spermatids $(1 n)$. We confirmed high purity (>90\%) for the pachytene spermatocyte preparation by immunostaining with anti- $\gamma \mathrm{H} 2 \mathrm{~A}$.X (Millipore) and anti-SCP3 (Abcam) antibodies. The round spermatid preparation was also almost homogeneous ( $>95 \%)$, as judged by immunostaining with anti-MVH antibody (Abcam).

\section{DNA methylation analysis}

Genomic DNA was prepared from cells using standard procedures (Sambrook and Russell 2001). For each cell type, DNA was isolated

\section{Genome Research www.genome.org}


from three or four mice. Genomic DNA was subjected to bisulfitemediated C-to-U conversion using BisulFast (Toyobo) and used as a template for PCR with ExTaq HS (Takara Bio). PCR comprised the following steps: (1) denaturation for $2 \mathrm{~min}$ at $95^{\circ} \mathrm{C}$; (2) 20 cycles of touchdown PCR (a first cycle for $15 \mathrm{sec}$ at $95^{\circ} \mathrm{C}$, for $15 \mathrm{sec}$ at $60^{\circ} \mathrm{C}$, and for $30 \mathrm{sec}$ at $72^{\circ} \mathrm{C}$; during subsequent cycles, the annealing temperature was decreased by $0.5^{\circ} \mathrm{C}$ at each cycle); and (3) 15 cycles of PCR for $15 \mathrm{sec}$ at $95^{\circ} \mathrm{C}$, for $15 \mathrm{sec}$ at $50^{\circ} \mathrm{C}$, and for $30 \mathrm{sec}$ at $72^{\circ} \mathrm{C}$. The PCR products purified from an agarose gel were cloned into the pGEM-Teasy vector (Promega), and at least 12 clones (typically 15-20 clones) were sequenced for each B1 locus in each cell type. PCR primers and amplicon sequences for the B1 loci are listed in Supplemental Table S1. PCR primers for the analysis of autonomous retroelements are listed in Supplemental Table S2. Their methylation was analyzed using the respective consensus sequences in Repbase (http://www.girinst.org/repbase/) as a guide.

\section{FISH analysis}

LINE probes were prepared by PCR using green-dUTP (Abbott Molecular), unmodified dNTPs, and mouse genomic DNA as a template. For SINE probes, we prepared probes for the B1 and B2 sequences using Cy3-dUTP (GE Healthcare) and mixed equivalent amounts of the B1 and B2 probes. The primers for LINE (L1) PCR were described previously (Solovei et al. 2009). The primers for B1 PCR (fragment size of $\sim 108 \mathrm{bp}$ ) were $5^{\prime}$-GTGGTGGCGCACGCCT$3^{\prime}$ and 5'-TAGCCCTGGCTGTCCTGGAA-3', and those for B2 PCR (fragment size of $\sim 151 \mathrm{bp}$ ) were $5^{\prime}$-GGCTGGTGAGATGGCTCAG3' and 5'-AGTACACTGTAGCTGTCTTCAGACAC-3'. Before use, the probes $(10 \mathrm{ng} / \mu \mathrm{L})$ were heat-denatured in $60 \%$ formamide, $2 \times$ SSC, and $50 \mathrm{mM}$ sodium phosphate (pH 7.0). Dual FISH with SINE and LINE probes was performed on frozen sections as follows: The testes from E15.5 embryos were directly embedded in OCT compound and frozen on dry ice. The samples were then sectioned $(6 \mu \mathrm{m})$ and fixed in $4 \%$ PFA. The complete hybridization protocol can be found at the following site: http://www.roche-applied-science. com/PROD_INF/MANUALS/InSitu/InSi_toc.htm. Germ cells were confirmed by immunostaining an adjacent section with anti-Oct3/4 antibody (Santa Cruz Biotechnology).

\section{RT-PCR analysis}

Total RNA was isolated from the spermatogonia of P7 mice (FACSsorted; three mice) and adult liver cells (three mice) using Isogene (Nippon Gene). cDNAs were synthesized from RNAs with random hexamers using a first-strand cDNA synthesis kit (Takara Bio) and used as a template for real-time PCR. The primer sequences are listed in Supplemental Table S3.

\section{Acknowledgments}

We thank Dr. Shin-ichi Tomizawa, Ms. Hatsune Chiba, Ms. Miho Miyake, and Mr. Yuichi Okudaira for their technical assistance. We also thank Drs. Yuji Kohara, Tomoko Tahira, and Hiroki Shibata for DNA sequencing. This work was supported by grants-in-aid to K.I. (22770009) and H.S. (20062010) from the Ministry of Education, Culture, Sports, Science and Technology of Japan, and by The Naito Foundation to K.I.

\section{References}

Aravin AA, Sachidanandam R, Girard A, Fejes-Toth K, Hannon GJ. 2007. Developmentally regulated piRNA clusters implicate MILI in transposon control. Science 316: 744-747.
Aravin AA, Sachidanandam R, Bourc'his D, Schaefer C, Pezic D, Toth KF, Bestor T, Hannon GJ. 2008. A piRNA pathway primed by individual transposons is linked to de novo DNA methylation in mice. Mol Cell 31: 785-799.

Bestor TH. 2000. The DNA methyltransferases of mammals. Hum Mol Genet 9: 2395-2402.

Bird A. 2002. DNA methylation patterns and epigenetic memory. Genes Dev 16: 6-21.

Bolzer A, Kreth G, Solovei I, Koehler D, Saracoglu K, Fauth C, Muller S, Eils R, Cremer C, Speicher MR, et al. 2005. Three-dimensional maps of all chromosomes in human male fibroblast nuclei and prometaphase rosettes. PLoS Biol 3: e157. doi: 10.1371/journal.pbio.0030157.

Bourc'his D, Bestor TH. 2004. Meiotic catastrophe and retrotransposon reactivation in male germ cells lacking Dnmt3L. Nature 431: 96-99.

Brennecke J, Aravin AA, Stark A, Dus M, Kellis M, Sachidanandam R, Hannon GJ. 2007. Discrete small RNA-generating loci as master regulators of transposon activity in Drosophila. Cell 128: 1089-1103.

Carmell MA, Girard A, van de Kant HJ, Bourc'his D, Bestor TH, de Rooij DG, Hannon GJ. 2007. MIWI2 is essential for spermatogenesis and repression of transposons in the mouse male germline. Dev Cell 12: 503 514.

Deininger PL, Batzer MA. 2002. Mammalian retroelements. Genome Res 12: 1455-1465.

Deininger PL, Moran JV, Batzer MA, Kazazian HH Jr. 2003. Mobile elements and mammalian genome evolution. Curr Opin Genet Dev 13: 651-658.

Edwards JR, O'Donnell AH, Rollins RA, Peckham HE, Lee C, Milekic MH, Chanrion B, Fu Y, Su T, Hibshoosh H, et al. 2010. Chromatin and sequence features that define the fine and gross structure of genomic methylation patterns. Genome Res 20: 972-980.

Elsik CG, Tellam RL, Worley KC, Gibbs RA, Muzny DM, Weinstock GM, Adelson DL, Eichler EE, Elnitski L, Guigo R, et al. 2009. The genome sequence of taurine cattle: A window to ruminant biology and evolution. Science 324: 522-528.

Guelen L, Pagie L, Brasset E, Meuleman W, Faza MB, Talhout W, Eussen BH de Klein A, Wessels L, de Laat W, et al. 2008. Domain organization of human chromosomes revealed by mapping of nuclear lamina interactions. Nature 453: 948-951.

Gunawardane LS, Saito K, Nishida KM, Miyoshi K, Kawamura Y, Nagami T, Siomi H, Siomi MC. 2007. A slicer-mediated mechanism for repeatassociated siRNA 5' end formation in Drosophila. Science 315: 15871590.

Hajkova P, Erhardt S, Lane N, Haaf T, El-Maarri O, Reik W, Walter J, Surani MA. 2002. Epigenetic reprogramming in mouse primordial germ cells. Mech Dev 117: 15-23.

Kaneda M, Okano M, Hata K, Sado T, Tsujimoto N, Li E, Sasaki H. 2004. Essential role for de novo DNA methyltransferase Dnmt3a in paternal and maternal imprinting. Nature 429: 900-903.

Kaplan G, Jelinek WR, Bachvarova R. 1985. Repetitive sequence transcripts and U1 RNA in mouse oocytes and eggs. Dev Biol 109: 15-24.

Kato Y, Kaneda M, Hata K, Kumaki K, Hisano M, Kohara Y, Okano M, Li E, Nozaki M, Sasaki H. 2007. Role of the Dnmt3 family in de novo methylation of imprinted and repetitive sequences during male germ cell development in the mouse. Hum Mol Genet 16: 2272-2280.

Kramerov DA, Vassetzky NS. 2005. Short retroposons in eukaryotic genomes. Int Rev Cytol 247: 165-221.

Kuramochi-Miyagawa S, Watanabe T, Gotoh K, Totoki Y, Toyoda A, Ikawa M, Asada N, Kojima K, Yamaguchi Y, Ijiri TW, et al. 2008. DNA methylation of retrotransposon genes is regulated by Piwi family members MILI and MIWI2 in murine fetal testes. Genes Dev 22: 908-917.

Kuramochi-Miyagawa S, Watanabe T, Gotoh K, Takamatsu K, Chuma S, Kojima-Kita K, Shiromoto Y, Asada N, Toyoda A, Fujiyama A, et al. 2010. $\mathrm{MVH}$ in piRNA processing and gene silencing of retrotransposons. Genes Dev 24: 887-892.

Lander ES, Linton LM, Birren B, Nusbaum C, Zody MC, Baldwin J, Devon K, Dewar K, Doyle M, FitzHugh W, et al. 2001. Initial sequencing and analysis of the human genome. Nature 409: 860-921.

Lane N, Dean W, Erhardt S, Hajkova P, Surani A, Walter J, Reik W. 2003. Resistance of IAPs to methylation reprogramming may provide a mechanism for epigenetic inheritance in the mouse. Genesis 35: 88-93.

Lees-Murdock DJ, De Felici M, Walsh CP. 2003. Methylation dynamics of repetitive DNA elements in the mouse germ cell lineage. Genomics 82: 230-237.

Li T, Spearow J, Rubin CM, Schmid CW. 1999. Physiological stresses increase mouse short interspersed element (SINE) RNA expression in vivo. Gene 239: 367-372.

Lindblad-Toh K, Wade CM, Mikkelsen TS, Karlsson EK, Jaffe DB, Kamal M, Clamp M, Chang JL, Kulbokas EJ III, Zody MC, et al. 2005. Genome sequence, comparative analysis and haplotype structure of the domestic dog. Nature 438: 803-819.

Ma L, Buchold GM, Greenbaum MP, Roy A, Burns KH, Zhu H, Han DY, Harris RA, Coarfa C, Gunaratne PH, et al. 2009. GASZ is essential for male 
meiosis and suppression of retrotransposon expression in the male germline. PLoS Genet 5: e1000635. doi: 10.1371/journal.pgen.1000635.

Matzke M, Kanno T, Huettel B, Daxinger L, Matzke AJ. 2007. Targets of RNAdirected DNA methylation. Curr Opin Plant Biol 10: 512-519.

Meissner A, Mikkelsen TS, Gu H, Wernig M, Hanna J, Sivachenko A, Zhang X, Bernstein BE, Nusbaum C, Jaffe DB, et al. 2008. Genome-scale DNA methylation maps of pluripotent and differentiated cells. Nature 454: 766-770.

Mikkelsen TS, Hillier LW, Eichler EE, Zody MC, Jaffe DB, Yang S, Enard W, Hellmann I, Lindblad-Toh K, Altheide TK, et al. 2005. Initial sequence of the chimpanzee genome and comparison with the human genome. Nature 437: 69-87.

Mikkelsen TS, Wakefield MJ, Aken B, Amemiya CT, Chang JL, Duke S, Garber M, Gentles AJ, Goodstadt L, Heger A, et al. 2007. Genome of the marsupial Monodelphis domestica reveals innovation in non-coding sequences. Nature 447: 167-177.

Ohnishi Y, Totoki Y, Toyoda A, Watanabe T, Yamamoto Y, Tokunaga K, Sakaki Y, Sasaki H, Hohjoh H. 2011. Active role of small non-coding RNAs derived from SINE/B1 retrotransposon during early mouse development. Mol Biol Rep. doi: 10.1007/s11033-011-0815-1.

Peaston AE, Evsikov AV, Graber JH, de Vries WN, Holbrook AE, Solter D, Knowles BB. 2004. Retrotransposons regulate host genes in mouse oocytes and preimplantation embryos. Dev Cell 7: 597-606.

Polak P, Domany E. 2006. Alu elements contain many binding sites for transcription factors and may play a role in regulation of developmental processes. BMC Genomics 7: 133. doi: 10.1186/14712164-7-133.

Popp C, Dean W, Feng S, Cokus SJ, Andrews S, Pellegrini M, Jacobsen SE, Reik W. 2010. Genome-wide erasure of DNA methylation in mouse primordial germ cells is affected by AID deficiency. Nature 463: 11011105 .

Reuter M, Chuma S, Tanaka T, Franz T, Stark A, Pillai RS. 2009. Loss of the Mili-interacting Tudor domain-containing protein-1 activates transposons and alters the Mili-associated small RNA profile. Nat Struct Mol Biol 16: 639-646.

Rodriguez J, Vives L, Jorda M, Morales C, Munoz M, Vendrell E, Peinado MA. 2008. Genome-wide tracking of unmethylated DNA Alu repeats in normal and cancer cells. Nucleic Acids Res 36: 770-784.

Sambrook J, Russell DW. 2001. Molecular cloning: A laboratory manual, 3rd ed. Cold Spring Harbor Laboratory Press, Cold Spring Harbor, NY.

Sasaki H, Matsui Y. 2008. Epigenetic events in mammalian germ-cell development: reprogramming and beyond. Nat Rev Genet 9: 129-140.

Seki Y, Hayashi K, Itoh K, Mizugaki M, Saitou M, Matsui Y. 2005. Extensive and orderly reprogramming of genome-wide chromatin modifications associated with specification and early development of germ cells in mice. Dev Biol 278: 440-458.

Shoji M, Tanaka T, Hosokawa M, Reuter M, Stark A, Kato Y, Kondoh G, Okawa K, Chujo T, Suzuki T, et al. 2009. The TDRD9-MIWI2 complex is essential for piRNA-mediated retrotransposon silencing in the mouse male germline. Dev Cell 17: 775-787.

Solovei I, Kreysing M, Lanctot C, Kosem S, Peichl L, Cremer T, Guck J, Joffe B. 2009. Nuclear architecture of rod photoreceptor cells adapts to vision in mammalian evolution. Cell 137: 356-368.

Tsirigos A, Rigoutsos I. 2009. Alu and B1 repeats have been selectively retained in the upstream and intronic regions of genes of specific functional classes. PLoS Comput Biol 5: e1000610. doi: 10.1371/ journal.pcbi.1000610.

Tsumura A, Hayakawa T, Kumaki Y, Takebayashi S, Sakaue M, Matsuoka C, Shimotohno K, Ishikawa F, Li E, Ueda HR, et al. 2006. Maintenance of self-renewal ability of mouse embryonic stem cells in the absence of DNA methyltransferases Dnmt1, Dnmt3a and Dnmt3b. Genes Cells 11: 805-814.

Walsh CP, Chaillet JR, Bestor TH. 1998. Transcription of IAP endogenous retroviruses is constrained by cytosine methylation. Nat Genet 20: 116117.

Warren WC, Hillier LW, Marshall Graves JA, Birney E, Ponting CP, Grutzner F, Belov K, Miller W, Clarke L, Chinwalla AT, et al. 2008. Genome analysis of the platypus reveals unique signatures of evolution. Nature 453: $175-183$.

Watanabe T, Chuma S, Yamamoto Y, Kuramochi-Miyagawa S, Totoki Y, Toyoda A, Hoki Y, Fujiyama A, Shibata T, Sado T, et al. 2011a. MitoPLD is a mitochondrial protein essential for nuage formation and piRNA biogenesis in the mouse germline. Dev Cell 20: 364-375.

Watanabe T, Tomizawa T, Mitsuya K, Totoki Y, Yamamoto Y, KuramochiMiyagawa S, Iida N, Hoki Y, Murphy PJ, Toyoda A, et al. 2011b. Role for piRNAs and a novel non-coding RNA in de novo DNA methylation of the imprinted mouse Rasgrf1 locus. Science 332: 848-852.

Waterston RH, Lindblad-Toh K, Birney E, Rogers J, Abril JF, Agarwal P, Agarwala R, Ainscough R, Alexandersson M, An P, et al. 2002. Initial sequencing and comparative analysis of the mouse genome. Nature 420: $520-562$.

Weber M, Hellmann I, Stadler MB, Ramos L, Paabo S, Rebhan M, Schubeler D. 2007. Distribution, silencing potential and evolutionary impact of promoter DNA methylation in the human genome. Nat Genet 39: 457466.

Xie H, Wang M, Bonaldo Mde F, Smith C, Rajaram V, Goldman S, Tomita T, Soares MB. 2009. High-throughput sequence-based epigenomic analysis of Alu repeats in human cerebellum. Nucleic Acids Res 37: 4331-4340.

Zheng K, Xiol J, Reuter M, Eckardt S, Leu NA, McLaughlin KJ, Stark A, Sachidanandam R, Pillai RS, Wang PJ. 2010. Mouse MOV10L1 associates with Piwi proteins and is an essential component of the Piwi-interacting RNA (piRNA) pathway. Proc Natl Acad Sci 107: 11841-11846.

Received March 23, 2011; accepted in revised form August 23, 2011. 
Genome Research 21: 2058-2066 (2011)

\section{Locus- and domain-dependent control of DNA methylation at mouse B1 retrotransposons during male germ cell development \\ Kenji Ichiyanagi, Yufeng Li, Toshiaki Watanabe, Tomoko Ichiyanagi, Kei Fukuda, Junko Kitayama, Yasuhiro Yamamoto, Satomi Kuramochi-Miyagawa, Toru Nakano, Yukihiro Yabuta, Yoshiyuki Seki, Mitinori Saitou, and Hiroyuki Sasaki}

The name of the second author was inadvertently misspelled in the author line of this article. Please note the correct spelling as Yufeng Li.

Genome Research 22: 51-63 (2012)

\section{Death of PRDM9 coincides with stabilization of the recombination landscape in the dog genome}

Erik Axelsson, Matthew T. Webster, Abhirami Ratnakumar, The LUPA Consortium, Chris P. Ponting, and Kerstin Lindblad-Toh

The authors have discovered an error in the formula to calculate GC*. In two instances, the definitions of $\mathrm{u}$ and $\mathrm{v}$ are reversed. The corrected text is as follows:

Page 57 (column 2, paragraph 2): Based on the assumption that the observed substitution patterns persist, we also estimate the equilibrium GC content $\left(\mathrm{GC}^{*} ; \mathrm{GC}^{*}=\mathrm{u} /[\mathrm{u}+\mathrm{v}]\right.$, where the rate of weak-to-strong [AT-to-GC] and strong-to-weak [GC-to-AT] substitutions are $u$ and v, respectively) (Meunier and Duret 2004) and predict that the GC peaks will be further reinforced in the dog but that they will vanish in the panda (Fig. 6).

Page 62 (column 1, paragraph 3): We calculated the average $\mathrm{GC}^{\star}\left(\mathrm{GC}^{\star}=\mathrm{u} /(\mathrm{u}+\mathrm{v})\right.$, where the rate of weak-tostrong [AT-to-GC] and strong-to-weak [GC-to-AT] substitutions are $\mathrm{u}$ and $\mathrm{v}$, respectively) in the dog as well as the panda, across the GC peak-centered, 18-kb windows described above using a 500-bp sliding window.

The authors apologize for any confusion this may have caused. 


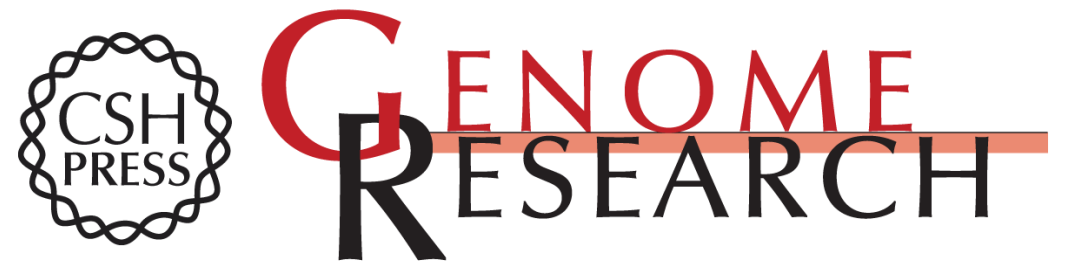

\section{Locus- and domain-dependent control of DNA methylation at mouse B1 retrotransposons during male germ cell development}

Kenji Ichiyanagi, Yungfeng Li, Toshiaki Watanabe, et al.

Genome Res. 2011 21: 2058-2066 originally published online October 31, 2011

Access the most recent version at doi:10.1101/gr.123679.111

Supplemental
Material http://genome.cshlp.org/content/suppl/2011/08/30/gr.123679.111.DC1

Related Content Errata

Genome Res. April , 2012 22: 810

References This article cites 50 articles, 10 of which can be accessed free at: http://genome.cshlp.org/content/21/12/2058.full.html\#ref-list-1

Articles cited in:

http://genome.cshlp.org/content/21/12/2058.full.html\#related-urls

\section{License}

Email Alerting Receive free email alerts when new articles cite this article - sign up in the box at the Service top right corner of the article or click here.

\section{Affordable, Accurate Sequencing.}

